\title{
FLASH MHD simulations of experiments that study shock-generated magnetic fields
}

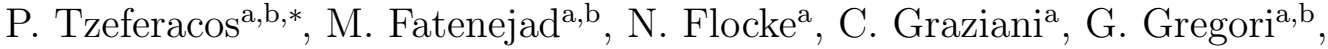

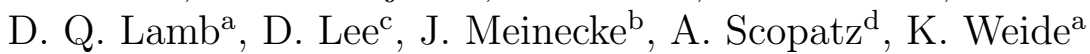 \\ ${ }^{a}$ The Flash Center for Computational Science, Department of Astronomy $\mathcal{E}$ \\ Astrophysics, University of Chicago, 5747 S. Ellis Av, Chicago, IL 60637, USA \\ ${ }^{b}$ Department of Physics, University of Oxford, Parks Road, Oxford OX1 3PU, UK \\ ${ }^{c}$ Department of Applied Mathematics \& Statistics, University of California-Santa Cruz, \\ 1156 High Street Mail Stop SOE2, Santa Cruz, CA 95064, USA \\ ${ }^{d}$ Department of Engineering Physics, University of Wisconsin-Madison, 1500 \\ Engineering Dr, Madison, WI 53706, USA
}

\begin{abstract}
We summarize recent additions and improvements to the high energy density physics capabilities in FLASH, highlighting new non-ideal magnetohydrodynamic (MHD) capabilities. We then describe 3D Cartesian and 2D cylindrical FLASH MHD simulations that have helped to design and analyze experiments conducted at the Vulcan laser facility. In these experiments, a laser illuminates a carbon rod target placed in a gas-filled chamber. A magnetic field diagnostic (called a Bdot) employing three very small induction coils is used to measure all three components of the magnetic field at a chosen point in space. The simulations have revealed that many fascinating physical processes occur in the experiments. These include megagauss magnetic fields generated by the interaction of the laser with the target via the Biermann battery mechanism, which are advected outward by the vaporized target material but decrease in strength due to expansion and resistivity; magnetic fields generated by an outward expanding shock via the Biermann battery mechanism; and a breakout shock that overtakes the first wave, the contact discontinuity between the target material and the gas, and then the initial expanding shock. Finally, we discuss the validation and predictive science we have done for this experiment with FLASH.
\end{abstract}

*PT petros.tzeferacos@flash.uchicago.edu 
Keywords: Magnetohydrodynamics, High energy density laboratory astrophysics, Numerical methods

\section{Introduction}

Magnetic fields, ubiquitously present in the universe, can play many and varied roles in astrophysical processes. The origin of these fields and the required mechanisms for their amplification to the observed values have been subjects of long-standing interest in astrophysics. One of the most promising explanations was put forward by [1], suggesting that the Biermann battery mechanism [2] could act in asymmetric shocks, formed during the hierarchical structure formation of proto-galaxies and halo mergers. The mechanism can produce a seed magnetic field - tiny in comparison with today's observed values — which could then be amplified via turbulent dynamo processes. Recently, this scenario has been put to the test in the context of laboratory experiments [3] performed at the LULI facility, capable of quantifying the seed field generation in laser-driven shocks.

The amplification of magnetic fields through turbulence is of great importance in supernova remnants. X-ray [4] and radio [5] observations of Cassiopeia A indicate magnetic fields that are almost a hundred times stronger than those in the surrounding interstellar medium. In the upwind region of the remnant, the flow is disorganized and is characterized by knots of radio synchrotron emission. The inferred magnetic field is of the order of a few milligauss, a value much larger than what shock compression would yield [6]. The reason behind this is probably connected to turbulent motions, generated either by hydrodynamic instabilities or, according to optical observations of Cassiopeia A, by the interaction of the ejecta with inhomogeneous and dense circumstellar clouds [7].

In a recent study [8], an international collaboration led by G. Gregori and the Oxford High Energy Density Laboratory Astrophysics (HEDLA) group managed to reproduce such processes in a controlled environment. A series of experiments were performed with the Vulcan laser at the Central Laser Facility of the Rutherford Appleton Laboratory: a laser illuminates a carbon rod target placed in a gas-filled chamber, launching a shock that generates magnetic field through the Biermann battery mechanism. This field is measured with a magnetic field diagnostic at a chosen point. The results indicate that the magnetic field is amplified, in particular when the shock interacts with a 
plastic grid that emulates the presence of density inhomogeneities found in an astrophysical environment. Using scaling relations [9], the experimental results can explain the observed synchrotron emission in the interior of the remnant.

In this collaboration, the Flash Center for Computational Science at the University of Chicago used the FLASH code $[10,11]$ to simulate the experiments, so as to obtain a better understanding of the physics in play and to explain the experimental results. FLASH$^{1}$ is an open-source, portable, modular, multi-physics, finite-volume, shock capturing code. The capabilities of the code include block-based Adaptive Mesh Refinement (AMR), sophisticated hydrodynamic and magneto-hydrodynamic (MHD) solvers, implicit solvers for diffusion, as well as an extensive range of algorithms for high energy density physics (HEDP), such as laser ray-tracing, radiation diffusion, and multi-material support with tabulated Equations of State (EoS) and opacities.

The goal of this article is two-fold: describe the HEDP extensions of the FLASH code mentioned above and apply the new code to model laboratory experiments that study the generation and turbulent amplification of magnetic fields in shocks. This article can be viewed as a companion paper to [8], providing further information about the computational campaign done in support of the experimental campaign. We will only consider the experimental shots in the absence of the plastic grid, so any turbulence that occurs is triggered by instabilities and not by an impulsive drive. Even so, the simulations have revealed that many fascinating physical processes occur in the experiments. For a more detailed discussion of the experiments and a full comparison with the numerical results, the reader should refer to [8].

This article is structured as follows: in section 2 we outline the HEDP capabilities of the FLASH code, as they were used in the simulation campaign; in section 3 we describe the experimental setup and the initial conditions for our runs; in section 4 we present a 3D Cartesian simulation that models the initial expansion of the blast wave; in section 5 we present $2 \mathrm{D}$ cylindrical results that follow the long-term evolution of the shock front to observable scales; in section 6 we discuss our results and outline future directions.

\footnotetext{
${ }^{1}$ publicly available at http://flash.uchicago.edu
} 


\section{High energy density physics with FLASH}

FLASH typically integrates systems of conservation laws of the general form

$$
\frac{\partial \mathbf{U}}{\partial t}+\nabla \cdot \mathbf{F}(\mathbf{U})=\mathbf{S}(\mathbf{U})
$$

where $\mathbf{U}$ denotes the conserved variables (e.g. $\mathbf{U} \equiv(\rho, \mathbf{m}, \mathbf{B}, \mathcal{E})^{T}$ for ideal MHD), $\mathbf{F}(\mathbf{U})$ the fluxes and $\mathbf{S}(\mathbf{U})$ the source terms. Here we have used the customary notation for density $(\rho)$, momentum density $(\mathbf{m})$, magnetic field (B) and total energy density $(\mathcal{E})$.

When modeling HEDP experiments, the usual single-temperature ideal MHD treatment is not sufficient, since there are a number of physical processes that cause departures from electron-ion-radiation thermal equilibrium, or which are critically affected by such disequilibria. Examples include shock and laser heating, transport coefficients, slow equilibration timescales, and radiation absorption and emission. In order to include these effects, we extend the standard MHD system of equations by assuming a singe-fluid treatment but separate temperatures for ions, electrons, and radiation (3T). This translates to the total pressure being defined as $p_{t o t}=p_{i}+p_{e}+p_{r}+\mathbf{B}^{2} / 2$, where the subscripts i, e, and $\mathrm{r}$ denote ions, electrons, and radiation, respectively. The continuity and momentum equations are given in their usual form

$$
\begin{gathered}
\frac{\partial \rho}{\partial t}+\nabla \cdot(\rho \mathbf{u})=0 \\
\frac{\partial \rho \mathbf{u}}{\partial t}+\nabla \cdot\left[\rho \mathbf{u u}+p_{t o t} \mathbf{I}-\mathbf{B B}\right]=0
\end{gathered}
$$

For the induction equation, on the other hand, we consider the generalized Ohm's law in the form $\mathbf{E}=-\mathbf{u} \times \mathbf{B}+\eta \mathbf{J}-\nabla p_{e} /\left(q_{e} n_{e}\right)$, where $q_{e}$ is the electron charge, $n_{e}$ the electron number density, $\eta$ the magnetic resistivity modeled as in [12], $\mathbf{J}=\nabla \times \mathbf{B}$ the current density and $\mathbf{E}$ the electric field. The induction equation then reads

$$
\frac{\partial \mathbf{B}}{\partial t}+\nabla \times[-\mathbf{u} \times \mathbf{B}]+\nabla \times\left[\eta \mathbf{J}-\frac{\nabla p_{e}}{q_{e} n_{e}}\right]=0
$$

where the last, non-ideal term considered on the left-hand side includes the magnetic diffusivity and the Biermann battery term. We also evolve the total energy density equation,

$$
\frac{\partial \mathcal{E}}{\partial t}+\nabla \cdot\left[\left(\mathcal{E}+p_{t o t}\right) \mathbf{u}-(\mathbf{u} \cdot \mathbf{B}) \mathbf{B}\right]-\nabla \cdot\left[\mathbf{B} \times\left(\eta \mathbf{J}-\frac{\nabla p_{e}}{q_{e} n_{e}}\right)\right]=-\nabla \cdot \mathbf{q}+S
$$


where the total energy density is given by $\mathcal{E}=\rho E_{\text {tot }}=\rho e_{\text {int }}+\rho \mathbf{u}^{2} / 2+\mathbf{B}^{2} / 2$ and the total specific internal energy includes the radiation energy, $e_{\text {int }}=$ $e_{i}+e_{e}+e_{r}$. We have also defined $\mathbf{q}=\mathbf{q}_{\mathbf{e}}+\mathbf{q}_{\mathbf{r}}$, the total heat flux, as the sum of the electron heat flux $\mathbf{q}_{\mathbf{e}}=-\kappa \nabla T_{e}$ and the radiation flux $\mathbf{q}_{\mathbf{r}}$. For the former, we set $\kappa$ the electron conductivity, and $T_{e}$ the electron temperature. The source term $S$ denotes external contributions of energy, typically due to laser heating.

In order to treat the $3 \mathrm{~T}$ components, we must also consider the nonconservative energy equations for electrons, ions, and radiation, which can be written as

$$
\begin{aligned}
\frac{\partial \rho e_{i}}{\partial t}+\nabla \cdot\left(\rho e_{i} \mathbf{u}\right)+p_{i} \nabla \cdot \mathbf{u}= & \rho \frac{c_{v, e}}{\tau_{e i}}\left(T_{e}-T_{i}\right) \\
\frac{\partial \rho e_{e}}{\partial t}+\nabla \cdot\left(\rho e_{e} \mathbf{u}\right)+p_{e} \nabla \cdot \mathbf{u}= & \rho \frac{c_{v, e}}{\tau_{e i}}\left(T_{i}-T_{e}\right)-\nabla \cdot \mathbf{q}_{e} \\
& +Q_{a b s}-Q_{e m i s}+Q_{l a s}+Q_{O h m} \\
\frac{\partial \rho e_{r}}{\partial t}+\nabla \cdot\left(\rho e_{r} \mathbf{u}\right)+p_{r} \nabla \cdot \mathbf{u}= & -\nabla \cdot \mathbf{q}_{r}-Q_{a b s}+Q_{e m i s}
\end{aligned}
$$

where $c_{v, e}$ is the electron specific heat, $\tau_{e i}$ is the ion-electron relaxation time, $Q_{a b s}$ denotes the rate of increase of the electron internal energy density due to radiation absorption, $Q_{\text {emis }}$ the respective decrease due to radiation emission and $Q_{O h m}$ the rate of increase of the electron internal energy density due to Ohmic heating. The system closes with $3 \mathrm{~T}$ equations of state that connect internal energies, temperatures, and pressures of the components. This is accomplished using either an analytical prescription or, more frequently, through tabulated EoS.

The system of equations 2-5 is a mixed hyperbolic-parabolic system. All the terms on the right-hand side of the equations are operator-split from the solution of the non-ideal single-fluid magneto-hydrodynamics. The latter is handled using the single-step, time marching algorithm of the unsplit staggered mesh (USM) [15, 16] for Cartesian coordinates and its extension to cylindrical systems [17]. Both resistivity and the Biermann battery term (considering also the algorithm discussed in [18]) are included in the staggered electric field, which allows us to preserve the solenoidality condition of B to machine accuracy through constrained transport.

In order to utilize the $3 \mathrm{~T} \mathrm{EoS} \mathrm{and} \mathrm{properly} \mathrm{distribute} \mathrm{the} \mathrm{update} \mathrm{of} e_{\text {int }}$ to its components, we need to advance the auxiliary equations 6-8. However, the work terms $p_{s} \nabla \mathbf{u}$ (with $s$ denoting ions, electrons, or radiation) are ill defined 
at shocks. To overcome this, we employ a method inspired by the radiationhydrodynamics code RAGE [19]. The method advances the advection portion of equations 6-8 and distributes the change due to work and total shockheating, recovered from the solution of equations 2-5, based on the pressure ratio of the components. This method correctly allocates energy to electrons and ions in smooth flows but erroneously allows for shock-heating of the electrons, thus over-predicting their temperature and underestimating the temperature of ions. In the limit of short equilibration timescales, however, the heat-exchange between the two species heals the difference and equalizes the temperatures.

The right-hand side of equations 6-8 is in turn operator-split and each physical process is handled separately. The first term on the right-hand side of equations 6 and 7 models the energy exchange between ions and electrons through collisions and is recovered by solving the system

$$
\begin{aligned}
\frac{\partial \rho e_{i}}{\partial t} & =\rho \frac{c_{v, e}}{\tau_{e i}}\left(T_{e}-T_{i}\right), \\
\frac{\partial \rho e_{e}}{\partial t} & =\rho \frac{c_{v, e}}{\tau_{e i}}\left(T_{i}-T_{e}\right) .
\end{aligned}
$$

The electron specific heat $c_{v, e}$ is recovered by the EoS and the ion-electron equilibration time is modeled as in [13],

$$
\tau_{e i}=\frac{3 k_{B}^{3 / 2}}{8 \sqrt{2 \pi} q_{e}^{4}} \frac{\left(m_{i} T_{e}+m_{e} T_{i}\right)^{3 / 2}}{\left(m_{e} m_{i}\right)^{1 / 2} \bar{z}^{2} n_{i} \ln \Lambda_{e i}},
$$

where $k_{B}$ is the Boltzmann constant, $m_{i}$ is the average mass of an ion, $m_{e}$ is the mass of electrons, $\bar{z}$ is the average ionization, $n_{i}$ is the ion number density and $\ln \Lambda_{e i}$ is the Coulomb Logarithm associated with ion-electron collision [14].

The second term on the right-hand side of eq. 7 represents the electron thermal conduction, which is solved implicitly using the HYPRE ${ }^{2}$ library on

$$
\rho \frac{\partial e_{e}}{\partial t}=\nabla \cdot \kappa \nabla T_{e}
$$

where the electron thermal conductivity is calculated as in $[13,20]$ :

$$
\kappa=\left(\frac{8}{\pi}\right)^{3 / 2} \frac{k_{B}^{7 / 2}}{q_{e}^{4} \sqrt{m_{e}}}\left(\frac{1}{1+3.3 / \bar{z}}\right) \frac{T_{e}^{5 / 2}}{\bar{z} \ln \Lambda_{e i}}
$$

\footnotetext{
${ }^{2}$ available at https://computation-rnd.llnl.gov/linear_solvers/software.php
} 
The implicit solve of eq. 12 is crucial to retain large time steps for typical values of $\kappa$. We also implement a flux limiter (see for example [21]) to prevent unphysically-large fluxes from occurring, in regions of large temperature gradients.

The HYPRE library is used also in the calculation of radiation diffusion: all terms on the right-hand side of eq. 8, some of which also appear in eq. 7, describe the radiation transport, which is handled using multi-group diffusion (MGD) approximation. FLASH divides the total radiation energy density into $N_{g}$ energy groups, where group $g$ is defined by the frequency range from $\nu_{g}$ to $\nu_{g+1}$ and the terms of interest can be defined as

$$
\begin{gathered}
\rho e_{r} \equiv u_{r}=\sum_{g=1}^{N_{g}} u_{g} \quad, \quad Q_{a b s}=\sum_{g=1}^{N_{g}} Q_{a b s, g} \\
Q_{e m i s}=\sum_{g=1}^{N_{g}} Q_{e m i s, g} \quad, \quad \mathbf{q}_{r}=\sum_{g=1}^{N_{g}} \mathbf{q}_{r, g} .
\end{gathered}
$$

We then solve the following system of equations, assuming that radiation is emitted in a Planck spectrum with a given emission opacity:

$$
\begin{aligned}
\frac{1}{c} \frac{\partial u_{g}}{\partial t} & -\nabla \cdot\left(\frac{1}{3 \sigma_{t, g}} \nabla u_{g}\right)+\sigma_{a, g} u_{g}=\sigma_{e, g} a T_{e}^{4} \frac{15}{\pi^{4}}\left[P\left(x_{g+1}\right)-P\left(x_{g}\right)\right] \\
\frac{\partial u_{e}}{\partial t} & =\sum_{g}\left\{\sigma_{a, g} u_{g}-\sigma_{e, g} a T_{e}^{4} \frac{15}{\pi^{4}}\left[P\left(x_{g+1}\right)-P\left(x_{g}\right)\right]\right\}
\end{aligned}
$$

where $\sigma_{t, g}$ is the transport opacity, $\sigma_{a, g}$ the absorption opacity, $\sigma_{e, g}$ the emission opacity, $a$ the radiation constant, and $P(x)$ the Planck integral. The argument to the Planck integral is $x=h \nu / k_{B} T_{e}$ where $h$ is Planck's constant. The second and third terms on the left-hand side of eq. 15 correspond to $\nabla \cdot \mathbf{q}_{r, g}$ and $Q_{a b s, g}$, respectively, while on the right-hand side we have $Q_{\text {emis,g}}$. As in the EoS, the opacities are recovered either using an analytic expression or interpolated from tables. For the radiation diffusion coefficient, we employ once more a flux limiter to limit the radiation flux to the free streaming limit.

The two remaining terms in eq. 7 are $Q_{o h m}$, and $Q_{\text {las }}$. The first represents the Ohmic heating and can be computed from the solution of the conserved system, whereas the second denotes the laser heating. The laser beams are modeled in the geometric optics approximation. Beams are comprised of a 


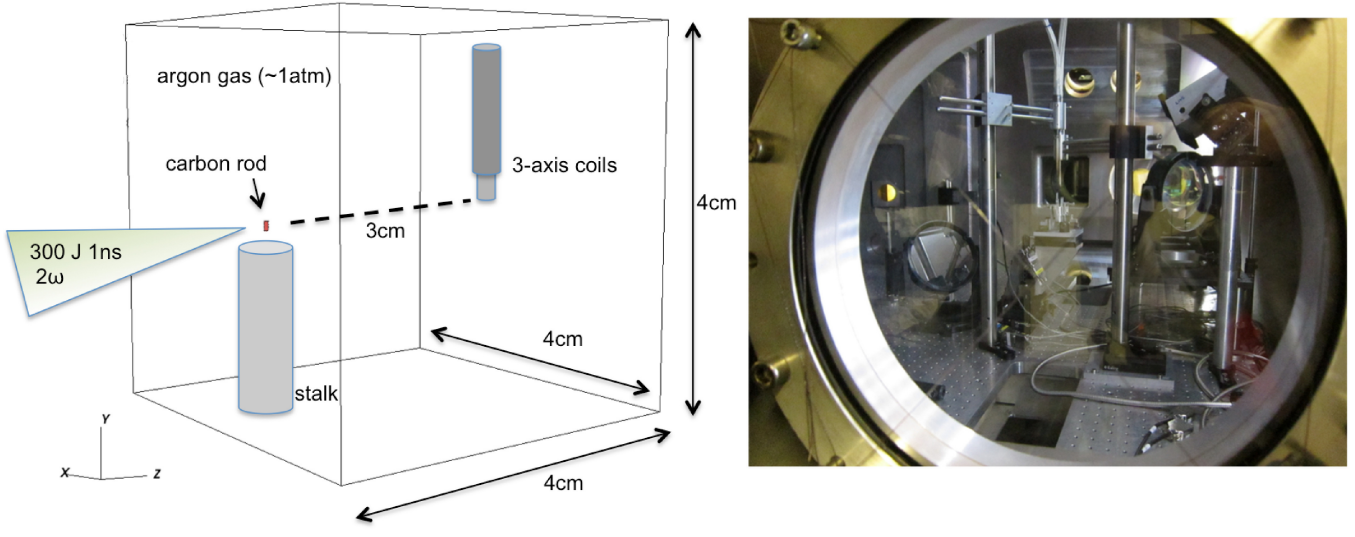

Figure 1: Left panel: Schematic of the initial configuration. A carbon rod, $500 \mu \mathrm{m}$ in diameter, is placed on a stalk inside a chamber filled with argon gas at $P \sim 1$ mbar. The rod is illuminated by three Vulcan beams. At a distance of $3 \mathrm{~cm}$ from the rod we situate the Bdot probe to measure the magnetic field signal. Notice that neither the stalk nor the Bdot probe are in scale. Right panel: A photograph through the porthole of the chamber. The experimental platform is located in the center. The crane holding the Bdot probe is also visible.

number of rays whose paths are traced through the computational domain based on the local refractive index of each cell. The laser power deposited in a cell is calculated based on the inverse bremsstrahlung power and depends on the local electron number density gradient and local electron temperature gradient. The ray tracing algorithm is based on [22]. More information regarding the above mentioned implementations, along with alternative algorithms, can be found in the FLASH code User's Guide ${ }^{3}$. The HEDP capabilities of the FLASH code have been recently exercised in a number of experiments, such as $[23,24]$, in addition to the experiments described next.

\section{Experimental configuration-initial conditions}

The experiment described in [8] is conducted inside a chamber filled with argon gas (Fig. 1), initially set at $P \sim 1 \mathrm{mbar}$ and room temperature. Three laser beams (527 nm wavelength) are focused onto a $500 \mu \mathrm{m}$ diameter carbon

\footnotetext{
${ }^{3}$ http://flash.uchicago.edu/site/flashcode/user_support
} 
rod, with a laser spot diameter of $300 \mu \mathrm{m}$. The total laser energy is $\sim 300 \mathrm{~J}$, delivered in a $1 \mathrm{~ns}$ pulse, with a corresponding intensity of $I=4 \times 10^{14} \mathrm{~W} / \mathrm{cm}^{2}$. The laser-target interaction launches a shock wave which propagates inside the gas. The evolution is monitored using transverse interferometry to recover electron densities and Schlieren imaging, which can measure density gradients. Three induction coils $[3,25]$ are placed at $3 \mathrm{~cm}$ from the carbon rod position. Each coil consists of four twisted pairs of coils wound around the axis of $\mathrm{a} \sim 1 \times 1 \mathrm{~mm}^{2}$ plastic core. The differential amplification of the voltage from the twisted pair loops cancels any voltage induced from the plasma's electric field components, and the magnetic field is calculated using Faraday's law. This allows an in situ measurement of the instantaneous rate of change of all three components of the magnetic field.

The experimental diagnostics follow the evolution the system for $\sim 10 \mu \mathrm{s}$. Combined with the large range in spatial scales, this makes the numerical modeling of the experiment quite challenging and the use of AMR crucial. Moreover, we need to employ all of the capabilities discussed in section 2 in order to properly model the physics processes in play. We have conducted a large number of 3D Cartesian and 2D cylindrical FLASH MHD simulations on BG/P and BG/Q supercomputers at Argonne National Laboratory with the support of the DOE Innovative and Novel Computational Impact on Theory and Experiment (INCITE) Program. The 3D runs can capture realistically the asymmetries in the produced shock wave and provide information on the early evolution of the system. The 2D simulations allow for much longer integration times, yielding results that can be compared directly to the experimental diagnostics.

\section{Three-dimensional results}

The three-dimensional computational box spans $4 \mathrm{~cm}$ in each direction, with $x$ and $y \in[-2 \mathrm{~cm}, 2 \mathrm{~cm}]$, whereas $z \in[-0.5 \mathrm{~cm}, 3.5 \mathrm{~cm}]$. The upper tip of the carbon rod is placed in the origin, its axis aligned with the $y$-axis. We use 6 levels of refinement, with an equivalent resolution of approximately $78 \mu \mathrm{m} /$ cell. The MGD solver assumes six energy groups, the boundaries of which are $0.1 \mathrm{eV}, 1 \mathrm{eV}, 10 \mathrm{eV}, 100 \mathrm{eV}, 1 \mathrm{KeV}, 10 \mathrm{KeV}$, and $100 \mathrm{KeV}$, while the tabulated opacities and EoS for carbon and argon are obtained with IONMIX4 [26]. We use a piecewise linear reconstruction with a minmod limiter, the Harten-Lax-van Leer (HLL) [27] Riemann solver, within the USM framework of [16]. Throughout the run we maintain a Courant-Friedrichs- 

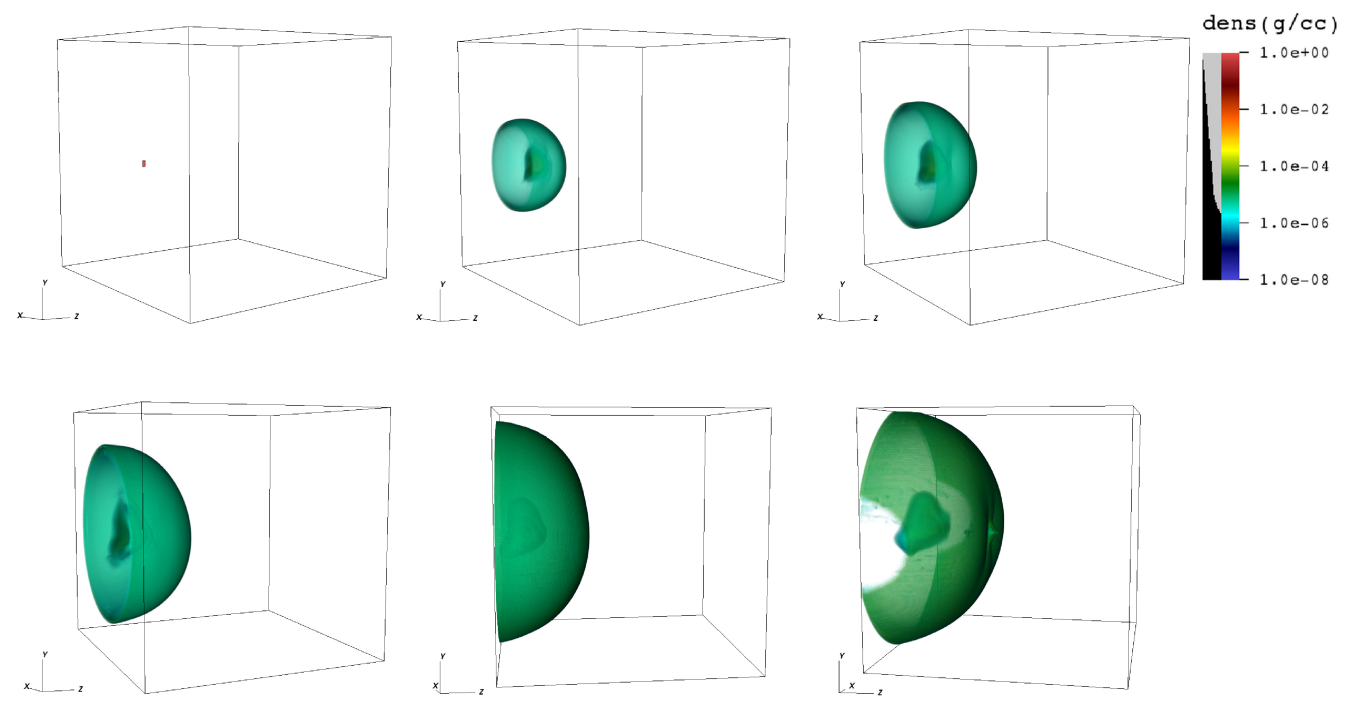

Figure 2: Temporal evolution of the laser-target interaction. We display from left to right the density logarithm at $t=0 \mathrm{~ns}, 10 \mathrm{~ns}, 20 \mathrm{~ns}$ (three upper panels) and $50 \mathrm{~ns}, 100 \mathrm{~ns}$, $150 \mathrm{~ns}$ (three lower panels). The shape of the shock in the last panel can be compared to the experimental image of figure $1 \mathrm{~b}$ in [8], where the front reaches the $Z=1 \mathrm{~cm}$ mark.
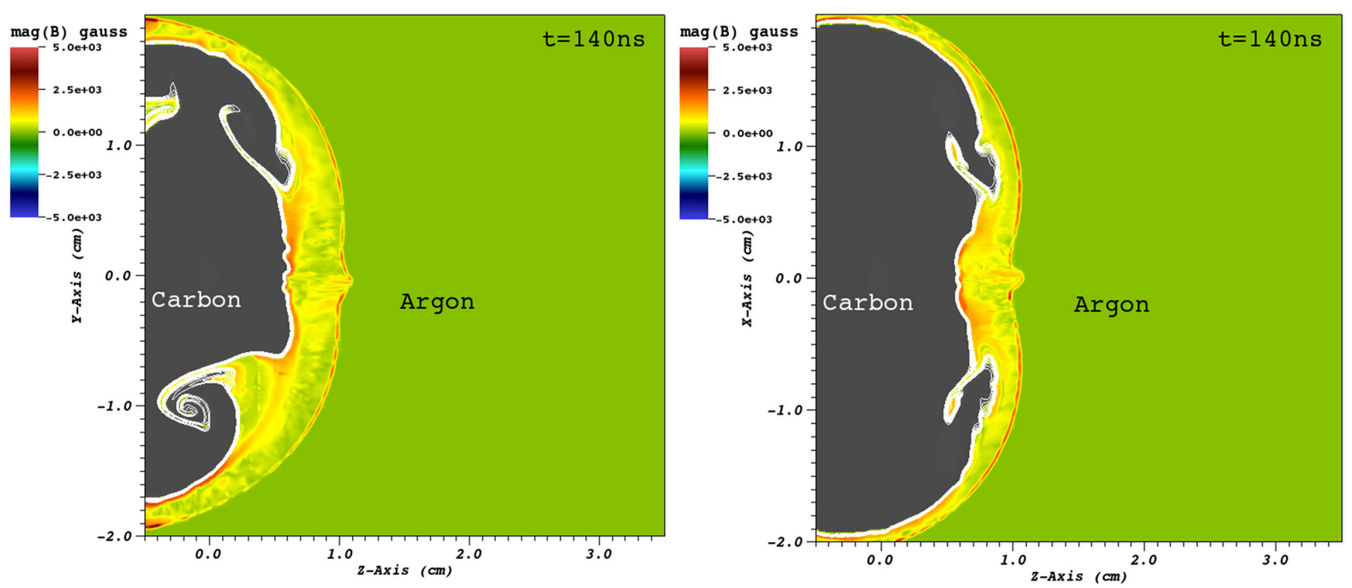

Figure 3: Two-dimensional slices in the $y$ - $z$ (left panel) and $x-z$ (right panel) planes. The color map displays the magnitude of the magnetic field in the Argon gas. The gray region shows the locus of the target material, and white contour lines depict the transition (mixing) region between argon and carbon; i.e., the material discontinuity. The snapshots are taken at $t=140 \mathrm{~ns}$. 
Lewy (CFL) number of 0.3. On all sides of the computational box we specify outflow (zero-gradient) conditions, except for the normal component of the staggered magnetic field which is recovered by the solenoidality condition.

We model a single $300 \mathrm{~J}, 2 \omega$ laser beam, aligned with the $z$-axis, whose spatial and temporal profile reproduce the specifications of the experiment. We illuminate the carbon rod close to its tip for $1 \mathrm{~ns}$ and follow the simulation's evolution for $180 \mathrm{~ns}$. In Fig. 2 we report stills of the density logarithm at different times in the evolution. The sequence of events is as follows:

- the laser initially ablates some target material on the back of the rod (at negative values of $\mathrm{z}$ );

- this launches a supersonic shock in the argon gas that promptly engulfs the rod, that is still mostly intact;

- the shock front is initially ballistic and enters a Sedov-Taylor phase at later times, as it sweeps up mass (outer front in the panels of Fig. 2);

- the ablated carbon also expands, remaining ballistic for longer due to the high density;

- the rocket-effect also launches a shock inside the carbon target, which manifests itself later on as a breakout shock, seen as the inner density feature in the late-time panels of Fig. 2.

Misalignment of electron pressure and electron density in the flow due to spatial inhomogeneities of the shock have as a result the generation of magnetic field through the Biermann battery mechanism, which can be written as

$$
\left.\frac{\partial \mathbf{B}}{\partial t}\right|_{\mathrm{BB}}=\nabla \times\left(\frac{\nabla p_{e}}{q_{e} n_{e}}\right)=\frac{\nabla p_{e} \times \nabla n_{e}}{q_{e} n_{e}^{2}} .
$$

Therefore, misalignment of electron pressure and electron density in the flow will generate magnetic field in the normal direction, downstream of the shock. This self-generated magnetic field is shown in Fig. 3. The two dimensional slices are taken on the $y-z$ and $x-z$ planes that intersect the origin. The obtained values are of the order of a kilogauss in the early phase of the evolution. These field values will however reduce in time due to expansion and magnetic diffusion, since the magnetic Reynolds number is small; i.e., of the order of a few. 

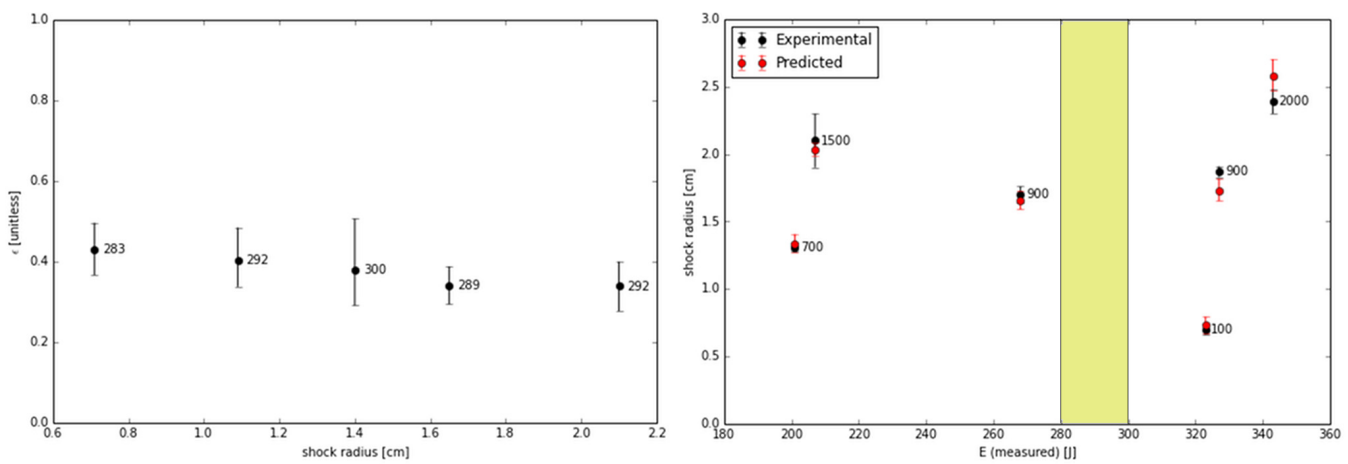

Figure 4: Left panel: Values of efficiency $\epsilon$ as a function of shock radius for the experimental shots used for calibration. The nominal laser energy is reported on each point in J. Right panel: Experimental versus predicted shock radii for an energy band $E_{e} \in[200-343 \mathrm{~J}]$. The yellow region shows the range in which the calibration was performed. The numbers reported on each point refer to the measurement's timing in ns.

The three-dimensional simulation reveals that the flow is highly asymmetric in the $y-z$ plane but fairly symmetric in the $x-z$. When comparing the two panels of Fig. 3 it is clear that the "side-view" of the left panel does not maintain top-bottom symmetry. Conversely, the "top-view" on the right exhibits reasonable right-left symmetry. This can be easily attributed to the alignment of the rod with the $y$-axis: as the expanding material engulfs the target, the flow surrounds the target in a symmetric fashion laterally, whereas it meets either the tip or the body of the rod while respectively moving upwards or downwards. This effect will be enhanced by the presence of the stalk that holds the target in place in the experimental setup, which is not included in the simulation (see for example Fig. 1 in [8]).

\section{Two-dimensional results}

In order to follow the evolution of the system for timescales that are comparable to those of the experiments, we now turn to a $2 \mathrm{D}$ cylindrical configuration. The two-dimensional computational box spans from 0 to $2 \mathrm{~cm}$ in the $R$ direction and from -1 to $3 \mathrm{~cm}$ in $Z$. The carbon rod is modeled as circular region at the origin. We use once more 6 levels of refinement, with an equivalent resolution of approximately $39 \mu \mathrm{m} /$ cell, and six energy groups for the MGD. The opacity and EoS tables are the same as those used in section 4 . The reconstruction is again a piecewise linear with a min- 
mod limiter, whereas we use the Harten-Lax-van Leer-Contact (HLLC) [28] Riemann solver, within the USM framework of [15] extended to cylindrical coordinates by [17]. We again specify outflow (zero-gradient) conditions on the sides of the computational box, except for the $Z$-axis where we assume axial symmetry.

We calibrated the fraction of the experimental laser energy that, when used in the simulation, matches the measured positions of the shock: we consider five different experimental shots for which the shock radius $\left(R_{e}\right)$ is calculated at a given time using Schlieren imaging data. The shots were chosen so that the range of experimental laser energies was small (280-300 J). For each of the shots, we perform simulations for five different fractions of an experimental laser energy of $300 \mathrm{~J}$ energy (namely, 0.2, 0.4, 0.6, 0.8, and 1 ), and calculate the numerically obtained shock radius.

Through interpolation, we calculate the effective energy $E_{\text {int }}$ needed in the numerical simulation to recover $R_{e}$, thus obtaining an efficiency value $\epsilon \equiv E_{\text {int }} / E_{e}$, where $E_{e}$ is the experimental laser energy (see the left panel of Fig. 4). From the five values we can now calculate a weighted mean efficiency $\hat{\epsilon}=0.37 \pm 0.08$. This value was then used to predict the shock radius for six other shots, with experimental energies spanning the range $200-343 \mathrm{~J}$. The predicted values of the shock radius agree with the experimental $R_{e}$ within error bars ${ }^{4}$ (right panel of Fig. 4).

Using the validated value of the energy efficiency, we now illuminate the carbon rod with $\hat{\epsilon} \times 300 \mathrm{~J}$ for $1 \mathrm{~ns}$. The single $2 \omega$ beam is aligned with the symmetry axis. We follow the evolution of the system for $4 \mu \mathrm{s}$, long enough to compare against the magnetic field signal from the Bdot probe (see fig. 3 of $[8]$ ).

The temporal evolution of the plasma density and the self-generated magnetic field is shown in Fig. 5. The early behavior of the flow agrees with our three-dimensional results, although the shock speed is considerably lower, matching the experimental data (right panel of Fig. 6). As in the 3D simulation, the laser-target interaction launches a supersonic shock in the argon gas, with carbon material expanding behind it (first three panels of Fig. 5).

\footnotetext{
${ }^{4}$ Note that the laser deposition efficiency is a standing problem in the laser-target interaction and the value of $\hat{\epsilon}$ that reproduces the data will change in different experimental setups (see for example [29] where $\hat{\epsilon} \simeq 1$ was used). A contributing factor for the relatively small value found in our calibration may be the attenuation of the inverse bremsstrahlung coefficient by the effects described in [30], not included in our runs.
} 

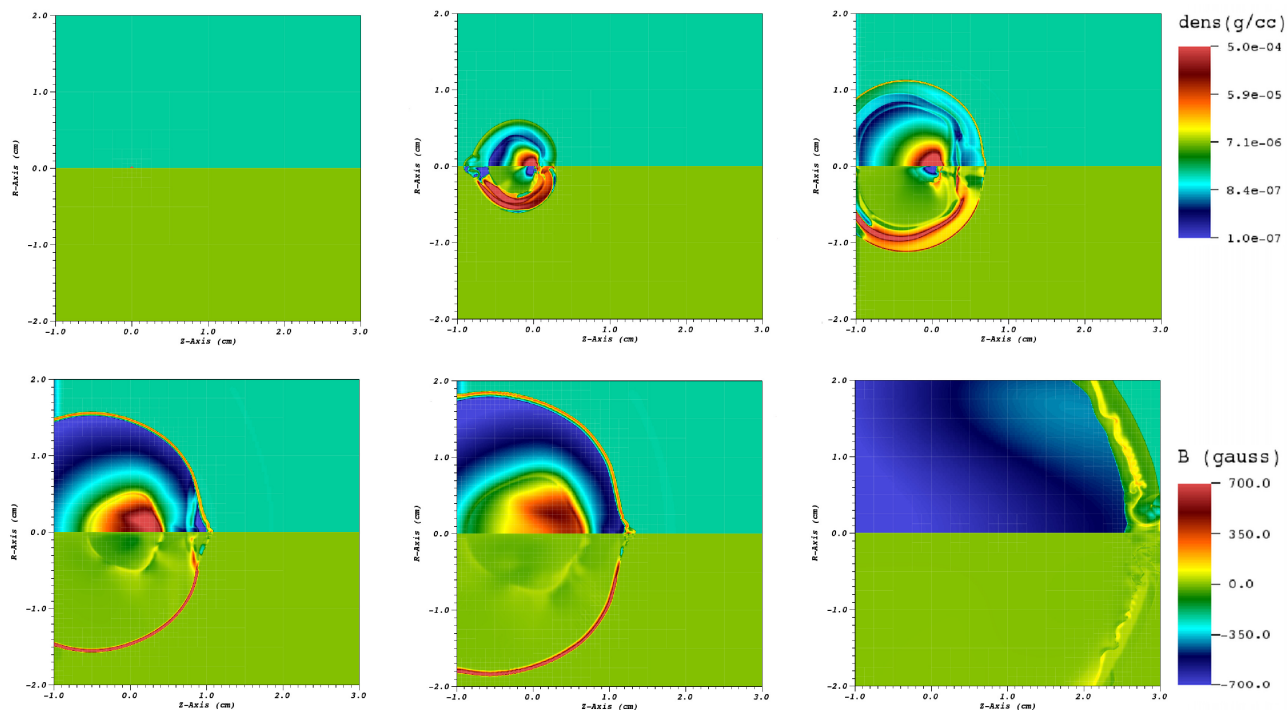

Figure 5: Temporal evolution of the laser-target interaction for the 2D cylindrical simulation. We display from left to right the density logarithm (top) and the magnetic field (bottom) at $t=0 \mathrm{~ns}, 20 \mathrm{~ns}, 100 \mathrm{~ns}$ (three upper panels) and $300 \mathrm{~ns}, 500 \mathrm{~ns}, 3 \mu \mathrm{s}$ (three lower panels). The semi-transparent white lines denote refinement levels.
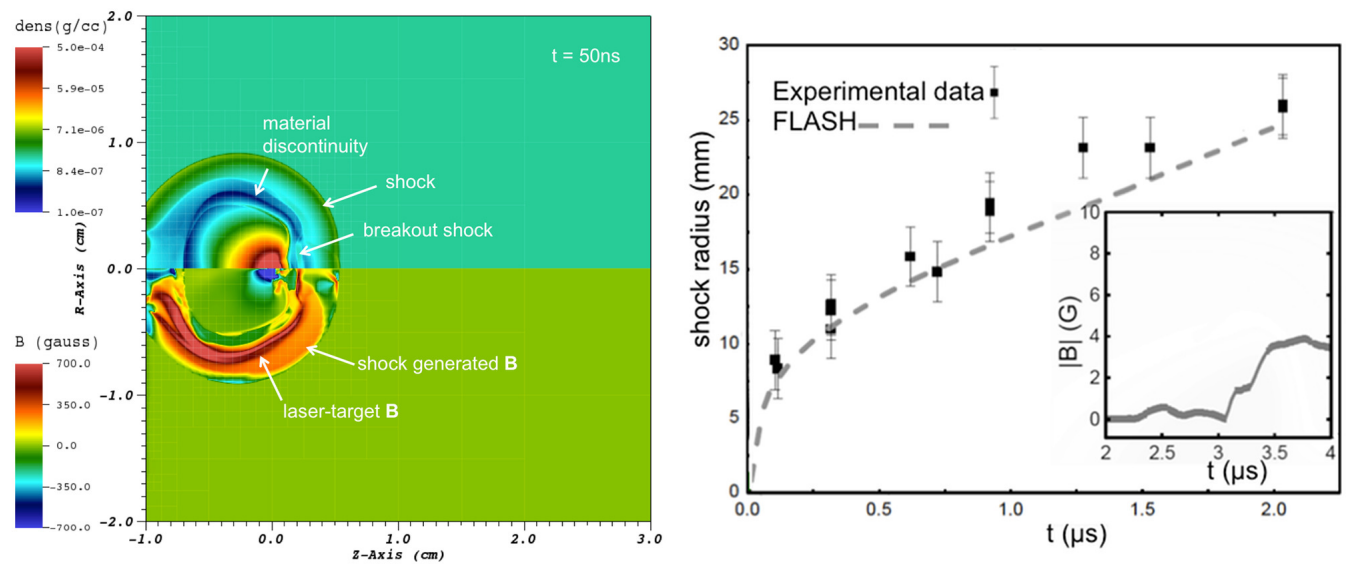

Figure 6: Left panel: Flow features in the density and magnetic field. The snapshot refers to $t=50$ ns. Right panel: Position-time diagram showing the experimental data overlayed on the numerical results. The inset reports the magnetic field time history calculated with FLASH. 
During the first few hundred ns we can distinguish three important features in the flow, namely the argon shock front, the material discontinuity separating carbon and argon, and a breakout shock traversing the target (see the left panel of Fig. 6). Due to pressure and density gradients misalignment (eq. 17), the flow generates magnetic field in the toroidal direction, normal to the computational plane. There are two apparent peaks in the field, a large one that is being advected outwards by the vaporized material, close to the material discontinuity, and a smaller one downstream of the argon shock.

As time progresses (fourth and fifth panel of Fig. 5), the outer shock enters a Sedov-Taylor phase and slows down, allowing for the material discontinuity to almost catch up, which results in the merging of the two magnetic field peaks to create a single feature of less than a kilogauss. At very late times, when the front approaches the location of the Bdot probe at $3 \mathrm{~cm}$, the dense breakout shock causes the material discontinuity to become RichtmyerMeshkov (RM) unstable [31]. By this time, the strength of the magnetic field is just a few gauss (see the last panel of Fig. 5).

As reported in [8], in order to emulate the Bdot signal we select a control volume of $3 \mathrm{~mm} \times 3 \mathrm{~mm}$ close to the $3 \mathrm{~cm}$ mark on the $Z$ axis where we average the generated field every $2 \mathrm{~ns}$, which is comparable to the time resolution of the Bdot probe $(10 \mathrm{~ns})$. The resulting time-series of magnetic field strength is reported in the inset of Fig. 6 and can be compared directly to the normal component of the field in the experiment, which is shown in red in the bottom right panel of fig. 3 in [8]. We find good quantitative agreement, both in terms of field strength and rise-time of the signal. This is also true for the remaining variables measured in the experiment, such as the peak electron density at the shock and the electron temperature. A detailed comparison with the experimental data and the connection of our results to astrophysical observations can be found in [8].

\section{Discussion}

In the first part of this article, we briefly outlined the HEDP-MHD capabilities implemented in the FLASH code and described the physical processes typically used in numerical modeling of laser-driven experiments. These include non-ideal MHD effects, such as the Biermann battery mechanism and magnetic resistivity, a three-temperature single-fluid approximation, thermal conduction, multi-group radiation diffusion, heat-exchange between electrons and ions, multi-material EoS and opacities, and laser energy deposition based 
on geometric ray-tracing. The code has been validated through benchmarks and code-to-code comparisons [32, 29], as well as through direct application to laboratory experiments $[8,23,24]$. We believe that the FLASH code can act as a useful open source tool for the academic HEDP community.

We then apply the HEDP capabilities of FLASH to model the laser-driven experiments performed with the Vulcan laser at the Central Laser Facility of the Rutherford Appleton Laboratory, in the context of an international collaboration led by G. Gregori and the Oxford High Energy Density Laboratory Astrophysics group. The results discussed in [8] show that asymmetric shocks produced by the laser-target interaction can generate magnetic fields which are amplified when the shock interacts with a plastic grid and becomes turbulent. Using scaling relations, the experimental results can be extrapolated to astrophysical scales and explain the strong fields observed downstream of supernova remnants such as Cassiopeia A.

Our simulation campaign supported the platform design and analysis of these experiments. We presented 3D Cartesian and 2D cylindrical, fullphysics simulations, that we used to study in detail the experiments performed without the plastic grid present. Even without the grid, the expanding plasma exhibits complex behavior, both in terms of flow characteristics and magnetic field. The 3D simulation allowed us to understand the early evolution, when the argon shock engulfs the target to form an asymmetric Sedov-Taylor blast wave; the ablated target material quickly expands behind the shock; and a second breakout shock traverses the rod, propagating outwards. The flow, especially its carbon component, is not top-bottom symmetric but maintains right-left symmetry fairly well. Using 2D cylindrical simulations, we then calibrated the fraction of the experimental laser energy that, when used in the simulation, matches the measured positions of the shock. With this calibration in hand, we performed an extended simulation to reach temporal scales comparable to the experimental diagnostics. We saw how at late times the material discontinuity catches up to the argon wave and eventually becomes RM unstable due to the breakout shock. Our simulation results agree well with all the experimentally measured quantities, including the magnetic field signal registered by the three-axis coils.

As a final note, we point out that both simulations and experiments discussed here and in [8] consider a plasma in which magnetic resistivity is particularly large, with a magnetic Reynolds number $\mathcal{R}_{m} \equiv v L / \eta \sim 5$ (see for example the supplementary table of [8]). This obviously limits the amplification of the magnetic field due to turbulence observed in the labora- 
tory, restricting the frequency spectrum of the magnetic energy to a Golitsyn power-law [33, 34] dependence, as shown in fig. 4 of [8]. In order to obtain non-linear turbulent amplification, as occurs in supernova remnants, we need significantly larger values of $\mathcal{R}_{m}$. The obvious solution is to increase the laser energy, as it would increase $v$ and decrease $\eta \propto T_{e}^{-3 / 2}$ by increasing the electron temperature. Alternative experimental setups could also produce larger magnetic Reynolds numbers. We are conducting FLASH simulations to devise experimental platforms that can achieve the desired plasma conditions.

\section{Acknowledgments}

The authors would like to thank the anonymous reviewer whose comments and suggestions have improved the exposition of this study. This work was supported in part at the University of Chicago by the U.S. Department of Energy (DOE) under contract B523820 to the NNSA ASC/Alliances Center for Astrophysical Thermonuclear Flashes; the Office of Advanced Scientific Computing Research, Office of Science, U.S. DOE, under contract DE-AC0206CH11357; the U.S. DOE NNSA ASC through the Argonne Institute for Computing in Science under field work proposal 57789; and the U.S. National Science Foundation under grant PHY-0903997. The research leading to these results has received funding from the European Research Council under the European Community's Seventh Framework Programme (FP7/2007-2013) / ERC grant agreement no. 256973. An award of computer time was provided by the DOE Innovative and Novel Computational Impact on Theory and Experiment (INCITE) program. This research used resources of the Argonne Leadership Computing Facility at Argonne National Laboratory, which is supported by the Office of Science of the U.S. Department of Energy under contract DE-AC02-06CH11357.

[1] R. M. Kulsrud, R. Cen, J. P. Ostriker, and D. Ryu, ApJ 480 (1997) 481.

[2] L. Biermann, Zeitschrift für Naturforschung Teil A 5 (1950) 65.

[3] G. Gregori et al., Nature 481 (2012) 480.

[4] U. Hwang, S. S. Holt, and R. Petre, ApJ 537 (2000) 119.

[5] M. C. Anderson, and L. Rudnick, ApJ 441 (1995) 307. 
[6] F. Guo, S. Li, H. Li, J. Giacalone, J. R. Jokipii, and D. Li, ApJ 747 (2012) 98.

[7] R. A. Chevalier, and J. Oishi, ApJ 593 (2003) L23.

[8] J. Meinecke et al., Nature Phys 10 (2014) 520.

[9] D. D. Ryutov, R. P. Drake, and B. A. Remington, ApJS 127 (2000) 465.

[10] B. Fryxell, et al., ApJS 131 (2000) 273.

[11] A. Dubey, K. Antypas, M. K. Ganapathy, L. B. Reid, K. Riley, D. Sheeler, A. Siegel, and K. Weide, Parallel Comp. 35 (2009) 512.

[12] S. I Braginskii, Rev. Plasma Phys. 1 (1965) 205.

[13] S. Atzeni, and J. Meyer-Ter-Vehn, (2004) The Physics of Inertial Fusion: Beam Plasma Interaction, Hydrodynamics, Hot Dense Matter (Oxford Science Publications).

[14] H. Brysk, P. M. Campbell, and P. Hammerling, Plasma Physics 17 (1974) 473.

[15] D. Lee, and A. E. Deane, J. Comput. Phys. 228 (2009) 952.

[16] D. Lee, J. Comput. Phys. 243 (2013) 269.

[17] P. Tzeferacos et al., HEDP 8 (2012) 322.

[18] M. Fatenejad et al., HEDP 9 (2013) 172.

[19] M. Gittings et al., Computational Science and Discovery 1 (2008) 015005.

[20] L. Spitzer, (1962) Physics of Fully Ionized Gases (New York: Wiley).

[21] D. Mihalas, and B. Weibel-Mihalas, (1999) Foundations of Radiation Hydrodynamics (New York: Dover).

[22] T. B. Kaiser, Phys. Rev. E 61 (2000) 895.

[23] K. Falk, E. J. Gamboa, G. Kagan, D. S. Montgomery, B. Srinivasan, P. Tzeferacos, and J. F. Benage, Phys. Rev. Lett. 112 (2014) 155003. 
[24] R. Yurchak et al., Phys. Rev. Lett. 112, 155001

[25] E. T Everson et al., Rev. Sci. Instrum. 80 (2009) 113505.

[26] J. J MacFarlane, Comp. Phys. Commun. 56 (1989) 259.

[27] A. Harten, P. D. Lax, and B. van Leer, SIAM Rev. 25 (1983) 35.

[28] S. Li, J. Comp. Phys. 203 (2005) 344.

[29] C. Orban, M. Fatenejad, S. Chawla, S. C. Wilks, D. Q. Lamb, arXiv:1306.1584 (2013).

[30] A. B. Langdon, Phys. Rev. Lett. 44 (1980) 575.

[31] M. Brouilette, Annu. Rev. Fluid Mech. 34 (2002) 445.

[32] M. Fatenejad, B. Fryxell, J. Wohlbier, E. Myra, D. Lamb, C. Fryer, and C. Graziani, HEDP 9 (2013) 63.

[33] G. S. Golitsyn, Soviet Phys. Doklady 5 (1960) 536.

[34] A. A. Schekochihin et al., New J. Phys. 9 (2007) 300. 